A three-frame digital image correlation (DIC) method for the measurement of small displacements and strains

This article has been downloaded from IOPscience. Please scroll down to see the full text article.

2012 Meas. Sci. Technol. 23105406

(http://iopscience.iop.org/0957-0233/23/10/105406)

View the table of contents for this issue, or go to the journal homepage for more

Download details:

IP Address: 213.246.216.140

The article was downloaded on 14/09/2012 at 09:38

Please note that terms and conditions apply. 


\title{
A three-frame digital image correlation (DIC) method for the measurement of small displacements and strains
}

\author{
C Cofaru ${ }^{1}$, W Philips ${ }^{1}$ and W Van Paepegem ${ }^{2}$ \\ ${ }^{1}$ Telin-IPI-IBBT, Ghent University, St-Pietersnieuwstraat 41, B-9000 Ghent, Belgium \\ 2 Department of Materials Science and Engineering, Ghent University, Technologiepark-Zwijnaarde 903, \\ B-9052 Zwijnaarde, Belgium \\ E-mail: Cornel.Cofaru@telin.ugent.be
}

Received 24 April 2012, in final form 16 August 2012

Published 14 September 2012

Online at stacks.iop.org/MST/23/105406

\begin{abstract}
Digital image correlation (DIC) has become a well-established approach for the calculation of full-field displacement and strains within the field of experimental mechanics. Since their introduction, DIC methods have been relying on only two images to measure the displacements and strains that materials undergo under load. It can be foreseen that the use of additional image information for the calculus of displacements and strains, although computationally more expensive, can positively impact DIC method accuracy under both ideal and challenging experimental conditions. Such accuracy improvements are especially important when measuring very small deformations, which still constitutes a great challenge: small displacements and strains translate into equally small digital image intensity changes on the material's surface, which are affected by the digitization processes of the imaging hardware and by other image acquisition effects such as image noise. This paper proposes a new three-frame Newton-Raphson DIC method and evaluates it from the standpoints of accuracy and speed. The method models the deformations that are to be measured under the assumption that the deformation occurs at approximately the same rate between each two consecutive images in the three image sequences that are employed. The aim is to investigate how the use of image data from more than two images impacts accuracy and what is the effect on the computational speed. The proposed method is compared with the classic two-frame Newton-Raphson method in three experiments. Two experiments rely on numerically deformed images that simulate heterogeneous deformations. The third experiment uses images from a real deformation experiment. Results indicate that although it is computationally more demanding, the three-frame method significantly improves displacement and strain accuracy and is less sensitive to image noise.
\end{abstract}

Keywords: digital image correlation, speckle image, sub-pixel accuracy, three-frame DIC (Some figures may appear in colour only in the online journal)

\section{Introduction}

Digital image correlation (DIC) methods have gained widespread adoption in the years following their introduction [1-4] as non-contact solutions for measuring full-field displacements and strains of objects under load. The basic principle on which the methods operate is that of finding correspondences between the pixels of an image showing the analyzed object before deformation (reference image) and one showing it after deformation (deformed image). Matching the pixels in the two images, and thus obtaining their relative displacements, is generally done by dividing the reference image into small blocks, also called subsets, of rectangular size, and searching for their best match in the 


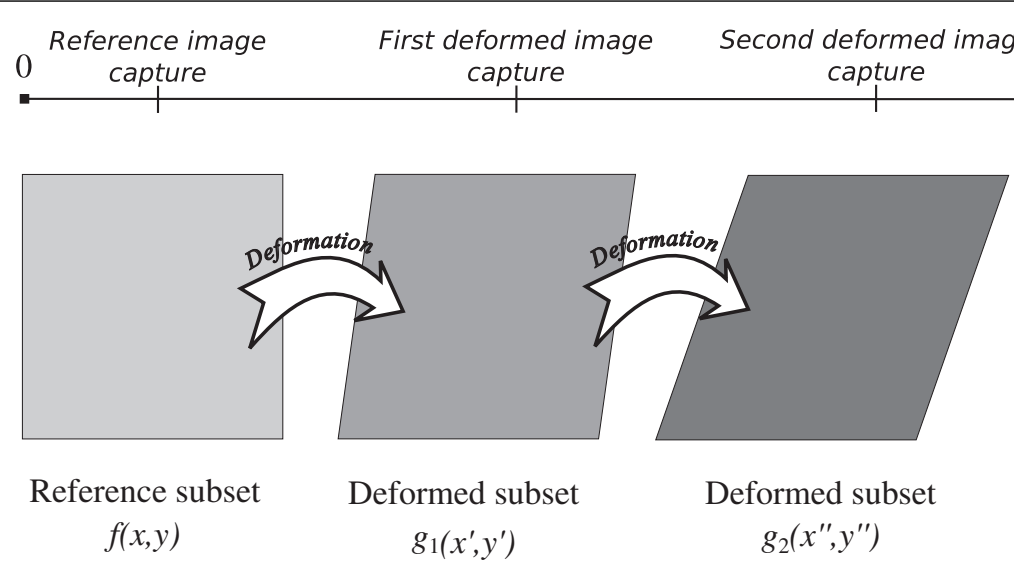

Figure 1. Three-frame DIC deformation assumption.

deformed image, optimizing a given similarity function. The analyzed objects are assumed to present a natural or applied random texture on their surface in order to solve the correspondence problem.

The estimation of motion using image sequences has received considerable attention with prevalence in the fields of video coding, processing and computer vision [5-16]. Two main benefits [7] of direct interest in the field of DIC can be identified: increased displacement accuracy and reduced noise sensitivity. Although substantial research has been directed towards improving or developing new DIC methods [17-28], very few approaches are adapted or have been specifically created to use more than two images to estimate deformations. The vast majority of DIC methods use only two images to calculate the displacements and strains. Solutions that integrate information spanning multiple images have been proposed in the form of spatio-temporal derivative filters [29] and by exploiting temporal image intensity variations at fixed coordinates throughout an image sequence [30].

The multi-frame DIC approach proposed here relies on the use of image sequences that contain three images. The deformation of a reference image is locally modelled at the subset level throughout the whole image sequence. The multiframe subset deformation model is built under the assumption that the object deformation that is to be measured occurs at approximately the same rate between each two consecutive images in the sequence. This principle can be extended to any number of frames, assuming each time that the deformation of a subset from one image to the next remains approximately constant. However, as the number of frames used increases, the underlying deformations can become more complex than the model. As will be seen later, the choice of three images suffices to illustrate the impact that the use of a greater number of images has on final strain estimates. The displacements and strains are calculated using a Newton-Raphson approach [18-20], which optimizes a quadratic similarity criterion that penalizes subset dissimilarities across the image sequence. The paper is structured as follows: section 2 contains the mathematical formulations of the proposed three-frame method; section 3 is devoted to the experimental details and results, while section 4 contains the conclusions.

\section{Three-frame Newton-Raphson formulation}

The proposed DIC method uses sequences of three images: by convention, we shall consider the first one as a reference image and the subsequent two as the deformed ones. The main assumption of the method, which is used to model subset deformations, is that locally the deformation between each two consecutive images occurs at the same rate and is relatively small. This means that a reference subset undergoes approximately the same deformation in the first deformed image as its corresponding deformed subset from the first deformed image in the second deformed image. The subset deformation assumption is graphically illustrated in figure 1 .

Such an assumption obviously imposes several constraints on the way the digital images are acquired during the experimental deformation process. In the case of the classic method, there are very few limitations with regard to the magnitude and complexity of the deformation contained in the deformed image. In this case, however, the deformations between the first two consecutive images and the next two have to be approximately the same, to satisfy the condition imposed by the subset deformation model. This is relatively easy to attain in experiments where the analysed material specimen deforms with relatively low speed. In experiments where deformations occur at high speed, the frame rates of the cameras must be increased accordingly. For high frame rates though, since more images have to be processed to characterize the material's behaviour under load, the computational complexity also increases. To determine the minimum frame rate that ensures the assumption is met, one could measure the displacements between pairs of consecutive frames, considering different frame rates: increasing the camera frame rate should lower displacement amplitudes and increase the likelihood of them being similar. The approach ultimately presents a trade-off: more data are used, inducing additional computational costs and increasing the complexity of experimental preparation procedures with the result that 
displacement and strain accuracy are improved. Regarding the amount of additional image information used, the three-frame method uses $50 \%$ more image data than the classic method. Alternatively, it can be stated that the classic method uses $66 \%$ of the three-frame method's available image data.

Let us consider the following: a reference subset $f(x, y)$ of size $N \times M$ pixels in the reference image and its corresponding, best matching two deformed subsets $g_{1}\left(x^{\prime}, y^{\prime}\right)$ and $g_{2}\left(x^{\prime \prime}, y^{\prime \prime}\right)$ of identical size in the two deformed images, respectively. The subset displacement model is set to be linear, with

$$
\begin{aligned}
& u(x, y)=p_{1}+p_{3}\left(x-x_{0}\right)+p_{5}\left(y-y_{0}\right), \\
& v(x, y)=p_{2}+p_{4}\left(x-x_{0}\right)+p_{6}\left(y-y_{0}\right),
\end{aligned}
$$

where $\left(x_{0}, y_{0}\right)$ are the coordinates of the centre of the subset. The unknown displacement component vector $\mathbf{p}=\left(p_{1}, \ldots, p_{6}\right)^{\top}$ is considered to characterize both the deformation between $f(x, y)$ and $g_{1}\left(x^{\prime}, y^{\prime}\right)$ and between $g_{1}\left(x^{\prime}, y^{\prime}\right)$ and $g_{2}\left(x^{\prime \prime}, y^{\prime \prime}\right)$ so that

$$
\begin{gathered}
f(x, y)=g_{1}(x, y ; \mathbf{p}), \\
g_{1}\left(x^{\prime}, y^{\prime}\right)=g_{2}\left(x^{\prime}, y^{\prime} ; \mathbf{p}\right) .
\end{gathered}
$$

Through the six displacement components, the deformation between $f(x, y)$ and $g_{2}\left(x^{\prime \prime}, y^{\prime \prime}\right)$ can also be modelled. The dependence between the pixel coordinates $x^{\prime \prime}, y^{\prime \prime}$ and $x, y$ has to be found in order to create a new subset displacement model, based on the assumed deformation between corresponding subsets in consecutive frames. This allows the introduction of an additional term in the optimization problem, besides the ones related to the subset pairs $f(x, y), g_{1}\left(x^{\prime}, y^{\prime}\right)$ and $g_{1}\left(x^{\prime}, y^{\prime}\right), g_{2}\left(x^{\prime \prime}, y^{\prime \prime}\right)$. Let us look at the relationships between the coordinates of pixels in the three subsets. First, we have

$$
\begin{aligned}
& x^{\prime}=x+p_{1}+p_{3}\left(x-x_{0}\right)+p_{5}\left(y-y_{0}\right), \\
& y^{\prime}=y+p_{2}+p_{4}\left(x-x_{0}\right)+p_{6}\left(y-y_{0}\right),
\end{aligned}
$$

between the coordinates of $f(x, y)$ and $g_{1}\left(x^{\prime}, y^{\prime}\right)$, and

$$
\begin{aligned}
& x^{\prime \prime}=x^{\prime}+p_{1}+p_{3}\left(x^{\prime}-x_{0}\right)+p_{5}\left(y^{\prime}-y_{0}\right), \\
& y^{\prime \prime}=y^{\prime}+p_{2}+p_{4}\left(x^{\prime}-x_{0}\right)+p_{6}\left(y^{\prime}-y_{0}\right),
\end{aligned}
$$

between the coordinates of $g_{1}\left(x^{\prime}, y^{\prime}\right)$ and $g_{2}\left(x^{\prime \prime}, y^{\prime \prime}\right)$. Introducing equations $(3 a)$ and $(3 b)$ into $(4 a)$ and $(4 b)$, we obtain

$$
\begin{aligned}
x^{\prime \prime}= & x+2 p_{1}+p_{1} p_{3}+p_{2} p_{5}+\left(2 p_{3}+p_{4} p_{5}+p_{3}^{2}\right)\left(x-x_{0}\right) \\
& +\left(2 p_{5}+p_{3} p_{5}+p_{5} p_{6}\right)\left(y-y_{0}\right), \\
y^{\prime \prime}= & +2 p_{2}+p_{1} p_{4}+p_{2} p_{6}+p_{4}\left(2+p_{3}+p_{6}\right)\left(x-x_{0}\right) \\
& +\left(2 p_{6}+p_{4} p_{5}+p_{6}^{2}\right)\left(y-y_{0}\right),
\end{aligned}
$$

respectively.
Here, an SSD-based subset similarity criterion $^{3}$ is employed to characterize subset similarity, which also includes the additional condition that the displacements of the pixels of $f(x, y)$ to $g_{2}\left(x^{\prime \prime}, y^{\prime \prime}\right)$ must conform to equations $(5 a)$ and $(5 b)$. The criterion is defined as

$$
\begin{aligned}
C_{\mathrm{SSD} 3 \mathrm{f}}(\mathbf{p}) & =\sum_{x, x^{\prime}=1}^{M} \sum_{y, y^{\prime}=1}^{N}\left[\left(f(x, y)-g_{1}(x, y ; \mathbf{p})\right)^{2}+\left(g_{1}\left(x^{\prime}, y^{\prime}\right)\right.\right. \\
& \left.\left.-g_{2}\left(x^{\prime}, y^{\prime} ; \mathbf{p}\right)\right)^{2}+\left(f(x, y)-g_{2}(x, y ; \mathbf{p})\right)^{2}\right]
\end{aligned}
$$

In the second term of the above equation, it can be observed that $\mathbf{p}$ is not present in the expression for $g_{1}$. The notation was adopted to show that $g_{1}$ is considered the reference subset with respect to $g_{2}$ and it is not deformed through interpolation in the iterative Newton-Raphson minimization process. Here, $g_{1}\left(x^{\prime}, y^{\prime}\right)$ represents the best match at the integer pixel level of $f(x, y)$ in the first deformed image. Consequently, in the minimization process, the subsets $g_{1}(x, y ; \mathbf{p}), g_{2}\left(x^{\prime}, y^{\prime} ; \mathbf{p}\right)$ and $g_{2}(x, y ; \mathbf{p})$ are iteratively warped through interpolation to match $f(x, y), g_{1}\left(x^{\prime}, y^{\prime}\right)$ and again, $f(x, y)$, respectively.

The three-frame method handles with relative robustness also the cases in which the consecutive subset deformations differ, contravening the assumed deformation model. Because a convex subset similarity criterion is used, the method will still converge to a solution that simultaneously optimizes the pixel differences in the three subset pairs from equation (6). The resulting subset displacement components $p_{i}$ will still characterize the deformations of $f(x, y)$ to $g_{1}\left(x^{\prime}, y^{\prime}\right)$ and of $g_{1}\left(x^{\prime}, y^{\prime}\right)$ to $g_{2}\left(x^{\prime \prime}, y^{\prime \prime}\right)$, although the two individual deformations are not the same. This can also be regarded as a form of temporal displacement component smoothing. In the tests performed here, differences of up to $30 \%$ between the underlying deformation of $f$ to $g_{1}$ and of $g_{1}$ to $g_{2}$ created no problems for the convergence of the method, considering the relatively low strain values used. In the case of larger strains, the maximum displacement component deviation admissible still requires more investigations.

Equation (6) is solved by setting its partial derivatives with respect to the displacement components to zero. The solution has the iterative form at the $t$ th iteration:

$$
\mathbf{p}^{(t)}=\mathbf{p}^{(t-1)}-\mathbf{H}_{C}^{-1}\left(\mathbf{p}^{(t-1)}\right) \mathbf{J}_{C}\left(\mathbf{p}^{(t-1)}\right),
$$

where $\mathbf{J}_{C}$ and $\mathbf{H}_{C}$ represent the Jacobian and Hessian matrices associated with $C_{\mathrm{SSD} 3 \mathrm{f}}$. The Jacobian and Hessian matrix elements are

$$
\begin{aligned}
& \frac{\partial}{\partial p_{i}} C\left(\mathbf{p}^{(t-1)}\right) \\
& =-\sum_{x=1}^{M} \sum_{y=1}^{N}\left[\frac{\partial}{\partial p_{i}} g_{1}\left(x, y ; \mathbf{p}^{(t-1)}\right)\left(f(x, y)-g_{1}\left(x, y ; \mathbf{p}^{(t-1)}\right)\right)\right] \\
& -\sum_{x^{\prime}=1}^{M} \sum_{y^{\prime}=1}^{N}\left[\frac{\partial}{\partial p_{i}} g_{2}\left(x^{\prime}, y^{\prime} ; \mathbf{p}^{(t-1)}\right)\left(g_{1}\left(x^{\prime}, y^{\prime}\right)-g_{2}\left(x^{\prime}, y^{\prime} ; \mathbf{p}^{(t-1)}\right)\right)\right] \\
& -\sum_{x=1}^{M} \sum_{y=1}^{N}\left[\frac{\partial}{\partial p_{i}} g_{2}\left(x, y ; \mathbf{p}^{(t-1)}\right)\left(f(x, y)-g_{2}\left(x, y ; \mathbf{p}^{(t-1)}\right)\right)\right]
\end{aligned}
$$

${ }^{3}$ For more simplicity and clarity, light intensity changes are not explicitly modelled in this criterion. These can be easily taken into account by adopting one of the approaches presented in [31]. 
and

$$
\begin{aligned}
& \frac{\partial^{2}}{\partial p_{i} \partial p_{j}} C\left(\mathbf{p}^{(t-1)}\right) \\
& =\sum_{x=1}^{M} \sum_{y=1}^{N}\left[\frac{\partial}{\partial p_{i}} g_{1}\left(x, y ; \mathbf{p}^{(t-1)}\right) \frac{\partial}{\partial p_{j}} g_{1}\left(x, y ; \mathbf{p}^{(t-1)}\right)\right] \\
& +\sum_{x^{\prime}=1}^{M} \sum_{y^{\prime}=1}^{N}\left[\frac{\partial}{\partial p_{i}} g_{2}\left(x^{\prime}, y^{\prime} ; \mathbf{p}^{(t-1)}\right) \frac{\partial}{\partial p_{j}} g_{2}\left(x^{\prime}, y^{\prime} ; \mathbf{p}^{(t-1)}\right)\right] \\
& +\sum_{x=1}^{M} \sum_{y=1}^{N}\left[\frac{\partial}{\partial p_{i}} g_{2}\left(x, y ; \mathbf{p}^{(t-1)}\right) \frac{\partial}{\partial p_{j}} g_{2}\left(x, y ; \mathbf{p}^{(t-1)}\right)\right]
\end{aligned}
$$

respectively, for $i, j=1, \ldots, 6$.

In the Jacobian and Hessian element expressions, the partial derivatives of $g_{1}(x, y ; \mathbf{p})$ and $g_{2}\left(x^{\prime}, y^{\prime} ; \mathbf{p}\right)$ with respect to the displacement components $p_{i}$ can be calculated as a function of the local image derivative values inside the deformed subsets $[19,20]$. The partial derivatives of $g_{2}(x, y ; \mathbf{p})$, which, according to the derivation chain rule, contain the partial derivatives $\partial x^{\prime \prime} / \partial p_{i}$ and $\partial y^{\prime \prime} / \partial p_{i}$ are determined as

$$
\begin{aligned}
\frac{\partial}{\partial p_{1}} g_{2}(x, y ; \mathbf{p}) & =\left(2+p_{3}\right) \frac{\partial}{\partial x^{\prime \prime}} g_{2}(x, y ; \mathbf{p}) \\
& +p_{4} \frac{\partial}{\partial y^{\prime \prime}} g_{2}(x, y ; \mathbf{p}) \\
\frac{\partial}{\partial p_{2}} g_{2}(x, y ; \mathbf{p}) & =p_{5} \frac{\partial}{\partial x^{\prime \prime}} g_{2}(x, y ; \mathbf{p}) \\
& +\left(2+p_{6}\right) \frac{\partial}{\partial y^{\prime \prime}} g_{2}(x, y ; \mathbf{p}) \\
& \left.+p_{5}\left(y-y_{0}\right)\right) \frac{\partial}{\partial x^{\prime \prime}} g_{2}(x, y ; \mathbf{p}) \\
& +p_{4}\left(x-x_{0}\right) \frac{\partial}{\partial y^{\prime \prime}} g_{2}(x, y ; \mathbf{p}) \\
\frac{\partial}{\partial p_{3}} g_{2}(x, y ; \mathbf{p}) & =\left(p_{1}+\left(2+2 p_{3}\right)\left(x-x_{0}\right)\right. \\
+\frac{\partial}{\partial p_{4}} g_{2}(x, y ; \mathbf{p})= & p_{5}\left(x-x_{0}\right) \frac{\partial}{\partial x^{\prime \prime}} g_{2}(x, y ; \mathbf{p}) \\
+ & \left(p_{1}+\left(2+p_{3}+p_{6}\right)\left(x-x_{0}\right)\right. \\
+ & \left.p_{5}\left(y-y_{0}\right)\right) \frac{\partial}{\partial y^{\prime \prime}} g_{2}(x, y ; \mathbf{p}) \\
+(x, y) & \\
+\mathbf{p})=\left(p_{4}(10\right. & \left.-y_{2}\right) \frac{\partial}{\partial y^{\prime \prime}} g_{2}(x, y ; \mathbf{p}) \\
g_{2}(x, y ; \mathbf{p})= & p_{5}\left(y-y_{0}\right) \frac{\partial}{\partial x^{\prime \prime}} g_{2}(x, y ; \mathbf{p}) \\
+ & p_{4}\left(x-x_{0}\right) \\
+ & \left.\left.p_{3}+p_{6}\right)\left(y-y_{0}\right)\right) \cdot \frac{\partial}{\partial y^{\prime \prime}} g_{2}(x, y ; \mathbf{p})
\end{aligned}
$$

In the implementation of the three-frame method, the reference image is divided into rectangular subsets and integer displacements are calculated through cross-correlation coefficient maximization. The best matching deformed subsets $g_{1}$ and $g_{2}$ for a reference subset $f$ at the integer pixel level are searched into the first and second deformed images. The integer pixel displacement between $g_{1}$ and $g_{2}$ is calculated as the difference between the integer displacement found between $f$ and $g_{2}$ and that found between $f$ and $g_{1}$ respectively. The method updates the whole horizontal and vertical displacement fields at each iteration. When all displacement components associated with the displacement of a subset vary by less than $10^{-5}$ in two consecutive iterations, convergence is considered to be reached and they will not be updated in subsequent iterations. Bi-cubic spline interpolation is used to numerically warp the deformed subsets towards their corresponding reference subsets at each iteration.

Spatial regularization introduces additional constraints into the subset similarity criterion with the aim of improving the spatial consistency or smoothness of the calculated displacements. The similarity criterion for the regularized three-frame method is obtained by adding a regularization term to the image data term from (6). The Geman-McClure robust estimator-based regularization introduced in [27] and later also used in [28] will be employed here. The associated robust shape parameters are calculated the same way as in [27]. The whole displacement field is updated at each iteration of the Newton-Raphson minimization process. Since the regularization term requires previously calculated neighbouring displacement components to be readily available at each iteration, its integration in the multi-frame methods is very straightforward.

\section{Experimental details and results}

\subsection{Experimental details}

The three-frame DIC method is evaluated using three (two numerical and one real) experiments along with the classic Newton-Raphson DIC method. In the evaluation, versions of the two methods that perform spatial motion regularization are also included. The behaviour of the proposed method in the presence of image noise is investigated as well. For this purpose, in one of the numerical experiments, random image noise with a normal distribution of zero mean and standard deviation of 3 is added to the images. The PSNRs calculated between the speckle images and their noisecorrupted counterparts are in the interval of 38.5-38.6 dB.

All deformed images in the numerical experiments are obtained by deforming a common $512 \times 512$ pixel reference image using the RBF interpolation framework introduced in [33]. The reference image is part of a $2208 \times 3000$ pixel 8-bit greyscale frame captured with a Pixelink PL-A782 camera that shows an aluminium specimen. The speckle pattern consists of an under-layer of white paint onto which black paint speckles have been sprayed. Through the camera calibration, the length of one image pixel corresponds to $8.33 \mu \mathrm{m}$ in the object plane. The displacements are based on the 'plate with hole' under biaxial stress displacement model [35], where the material simulated is rubber with $E=0.1 \mathrm{GPa}, v=0.5$ and the radius 


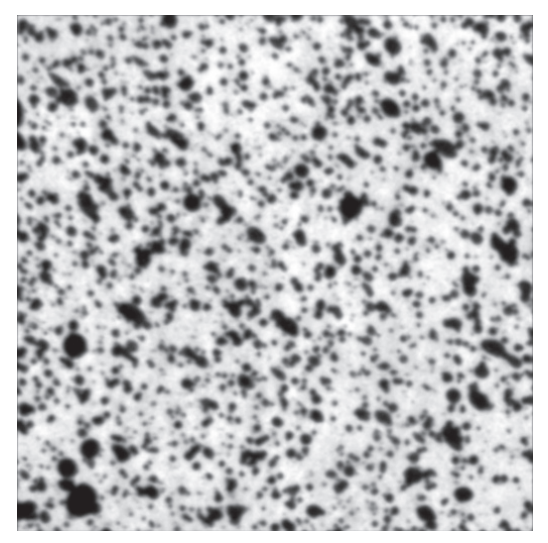

(a)

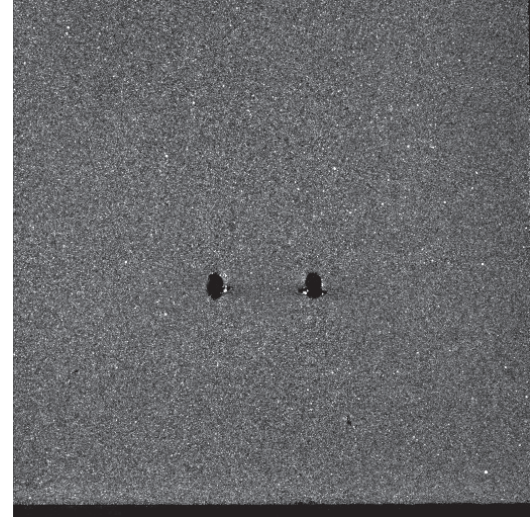

$(b)$

Figure 2. Examples of speckle images used in the multi-frame DIC method evaluation: $(a)$ the reference image for the two numerical experiments; $(b)$ the 285th image in the real image sequence.

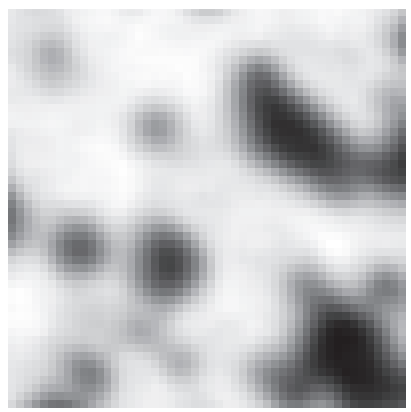

(a)

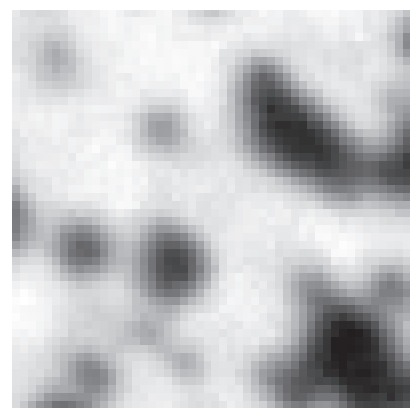

(b)
Figure 3. Detail of the reference image used in the numerical evaluation: $(a)$ original; $(b)$ with the addition of normally distributed image noise. of the hole is 50 pixels. The two numerical experiments can be summarized as follows.

- In the first experiment, the deformed images simulate deformations corresponding to vertically applied stresses of 0.1 and $0.2 \mathrm{MPa}$, respectively. The first deformed image (simulating the vertical stress of $0.1 \mathrm{MPa}$ ) presents artificial horizontal displacements ranging from -0.14 to 0.14 pixels. Vertical displacements are between -0.28 and 0.28 pixels. Horizontal and shear (absolute) strain values are smaller than $10^{-3}$. Variations between $1.6 \times 10^{-4}$ and $2.2 \times 10^{-3}$ are present for $\varepsilon_{y y}$. In the second deformed image (that simulates the vertical stress of $0.2 \mathrm{MPa}$ ), displacements and strains are twice as large compared to those from the first deformed image (the displacements
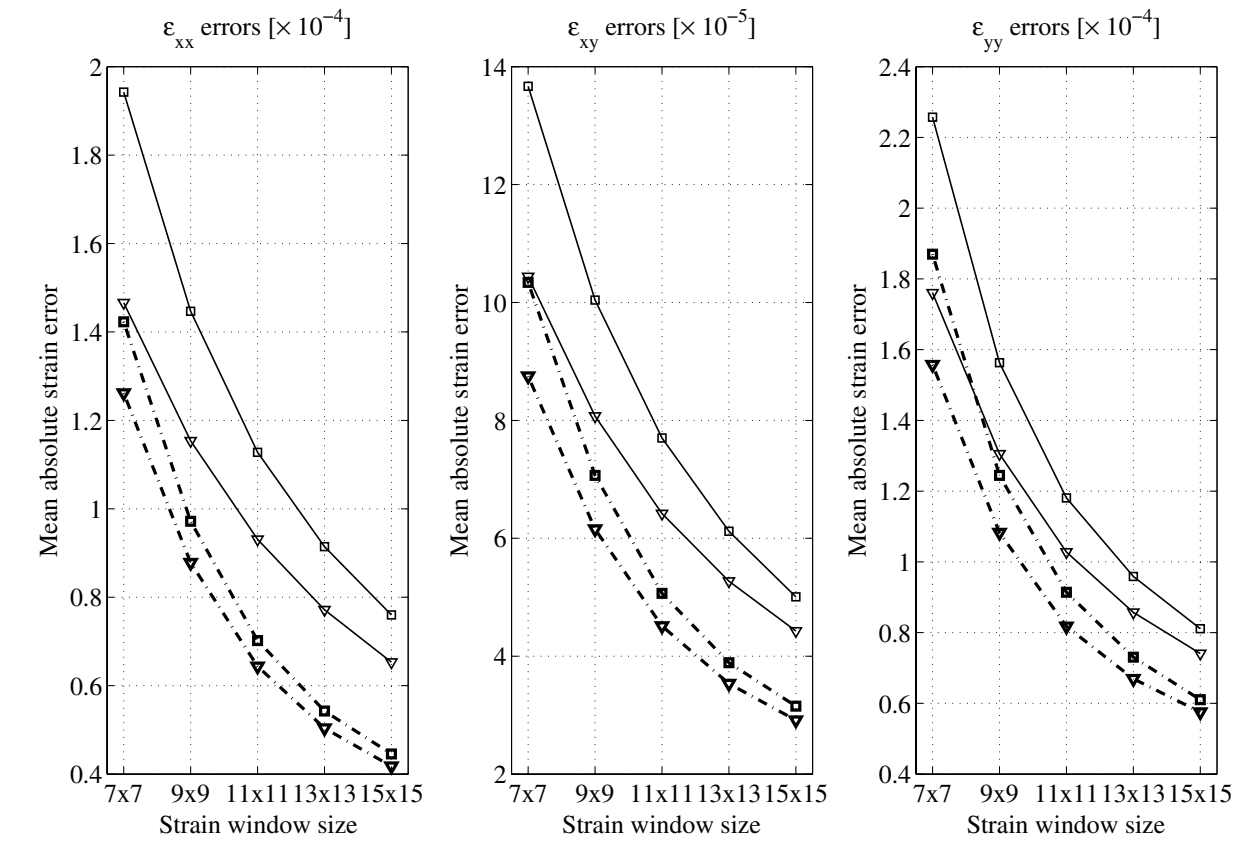

$\square-$ Classic N-R $\rightarrow-$ Classic N-R + regularization $\cdot-\square-\cdot 3-$ frame based N-R $-\nabla-\cdot 3-$ frame based N-R + regularization

Figure 4. Mean absolute strain errors for the first experiment, $21 \times 21$ pixel subsets. 

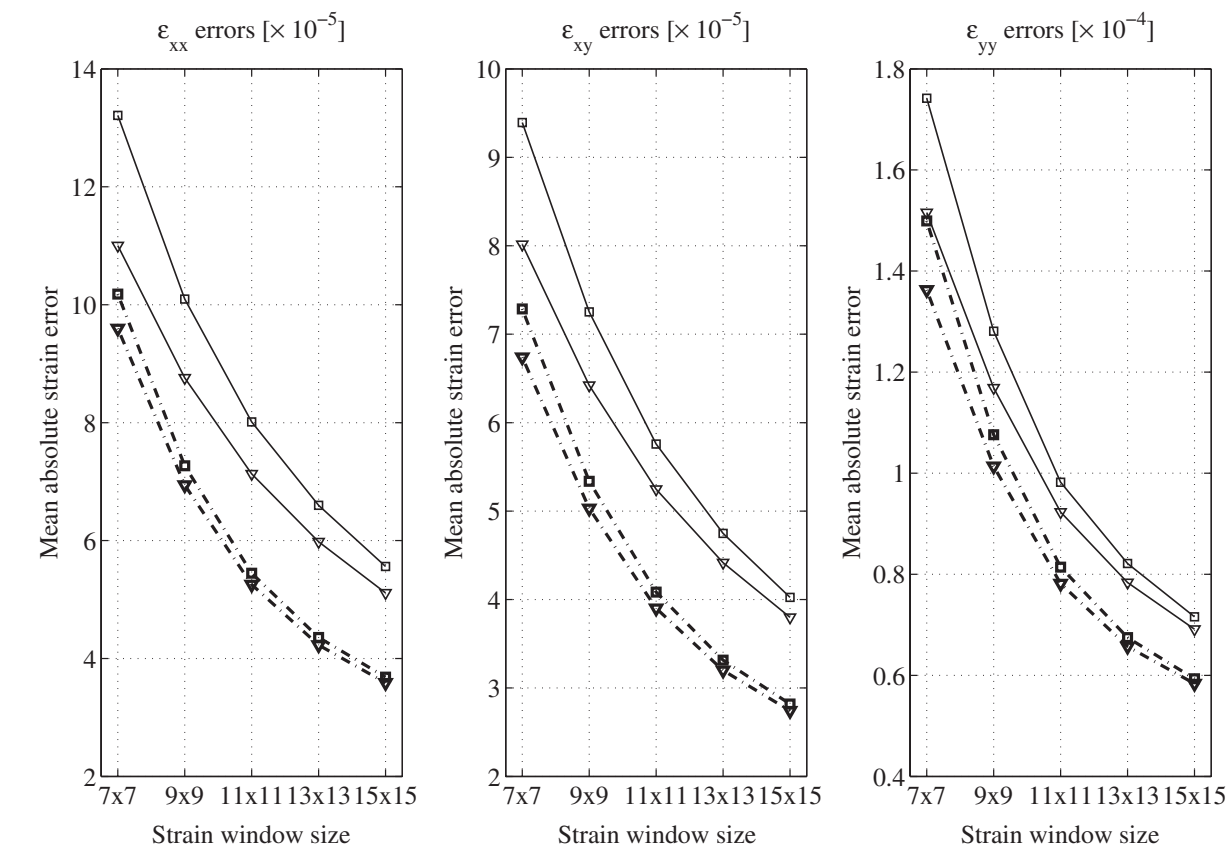

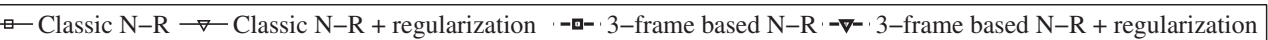

Figure 5. Mean absolute strain errors for the first experiment, $25 \times 25$ pixel subsets.
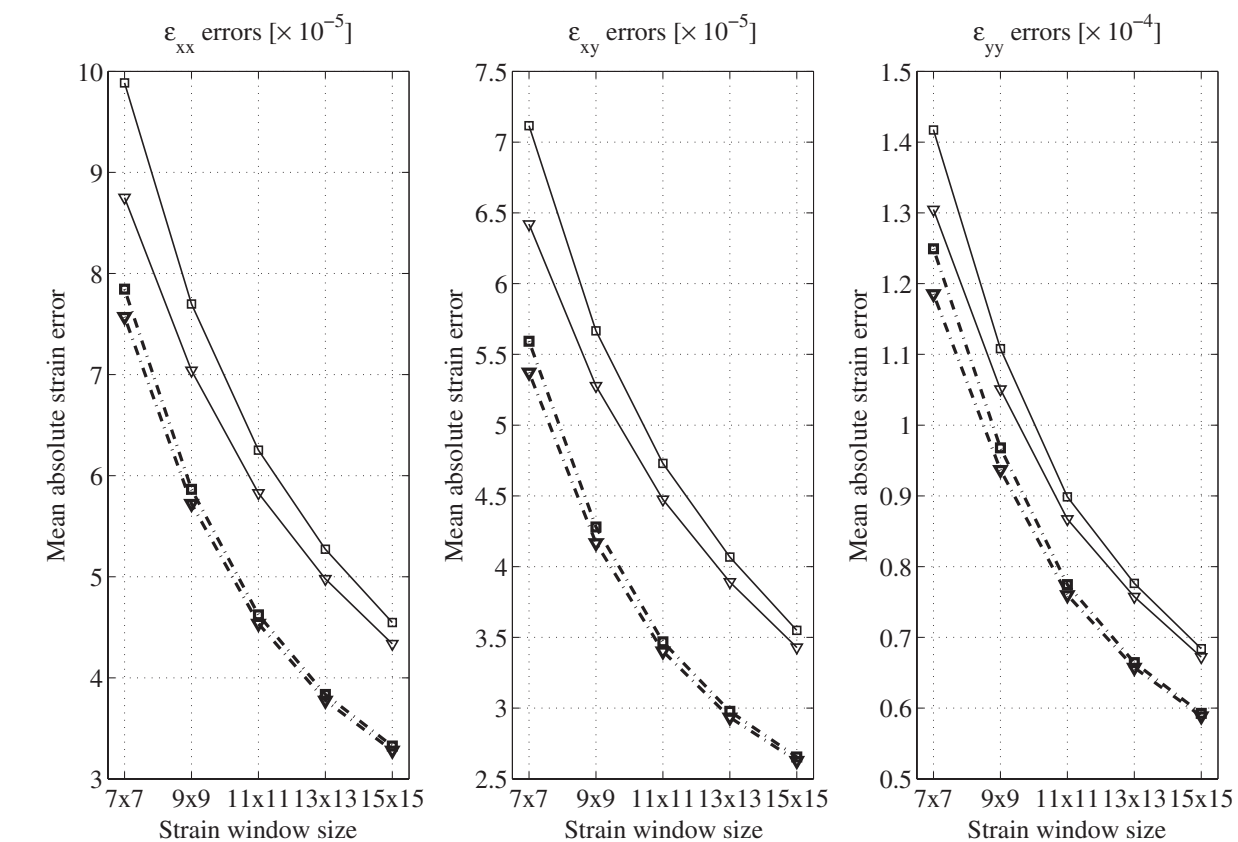

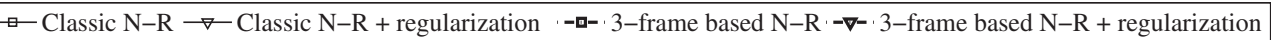

Figure 6. Mean absolute strain errors for the first experiment, $29 \times 29$ pixel subsets.

and implicitly strains for the 'plate with hole' model are in direct proportion to the applied stresses). Horizontal displacements vary between -0.28 and 0.28 pixels, vertical displacements vary between -0.56 and 0.56 pixels. The strains show variations between $-1.9 \times 10^{-3}$ and $-2.6 \times 10^{-4}$ for $\varepsilon_{x x},-1.6 \times 10^{-3}$ and $1.6 \times$ $10^{-3}$ for $\varepsilon_{x y}$ and between $3.2 \times 10^{-4}$ and $4.4 \times 10^{-3}$ for $\varepsilon_{y y}$;
- The second experiment presents identical deformations to the ones in the first experiment, with the difference that image noise is added to all three images.

The images used in the real experiment come from an experiment in which a thin polyester glass security film is deformed [34]. The film presents two holes, close to one another in its central area. To obtain the speckle pattern, the surface of the film is sprayed with a matt black paint, 

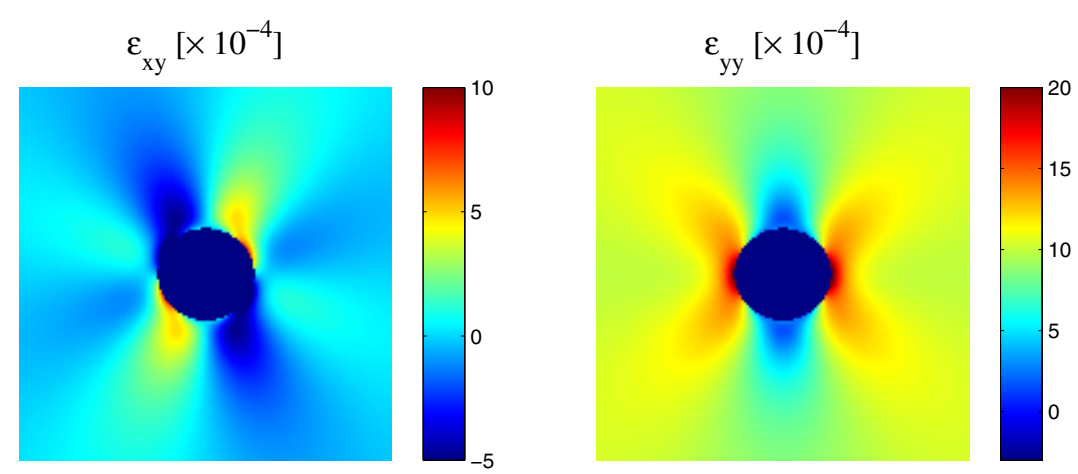

(a)
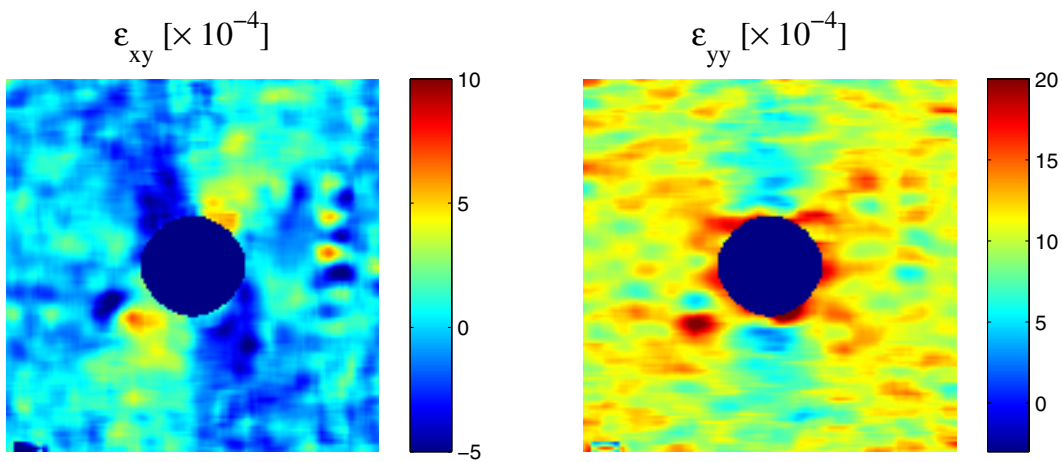

(b)
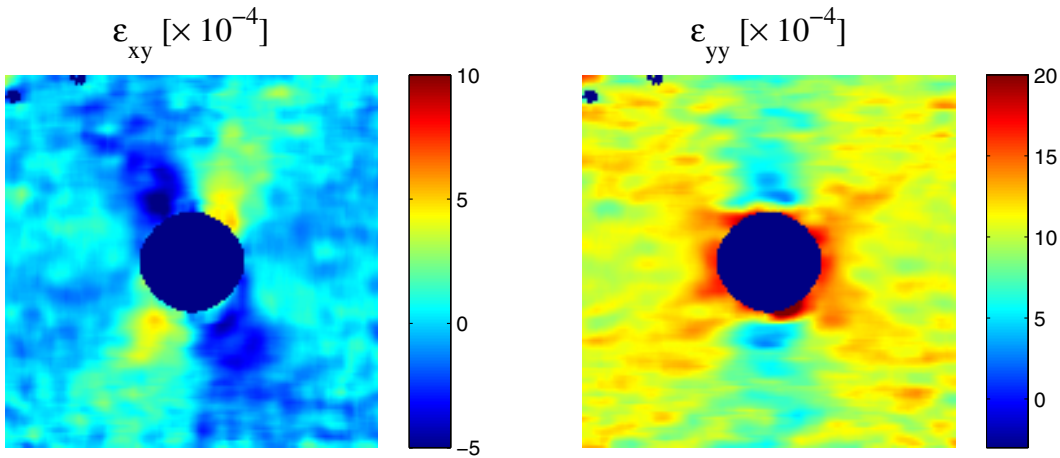

$(c)$

Figure 7. Shear (left column) and vertical (right column) strains corresponding to the first experiment: $(a)$ theoretical strains; $(b)$ strains calculated through the classic Newton-Raphson DIC method; $(c)$ strains calculated through the three-frame DIC method (using also spatial regularization). The subset size was $21 \times 21$ pixels and the strain window size was $11 \times 11$ displacements.

followed by a very fine dust of white matt paint. The chosen paint adheres to the film surface and provides a nonflaking thin layer that does not change the properties of the film. To illuminate the analysed object, two slide projectors with $100 \mathrm{~W}$ halogen bulbs and integrated infrared filters reducing the unwanted thermal spectrum were used. These were positioned symmetrically with respect to the optical axis (which is perpendicular to the film's surface). The specimen was photographed during deformation at a frame rate of $4 \mathrm{fps}$ with a Hitachi P110 CCD camera at a resolution of $1024 \times$ 1024 pixels and 8-bit greyscale intensity quantization. Also, a one pixel displacement in the image plane corresponds to a $23.8 \mu \mathrm{m}$ displacement in the object plane. The resulting speckle pattern contained densely packed, small speckles, of maximum 5 pixels in diameter. Noise levels are low throughout the images. The specimen was stretched by means of an especially designed low vibration hydraulic loading device. In total, 297 images are captured before the specimen ruptures due to the sustained load.

- The third experiment is used to provide an experimental validation of the proposed method. The reference and two deformed images correspond to the 285-, 286- and 287 th images in the sequence showing the deformation of the polyester glass security film. The horizontal and vertical displacements present between each two consecutive images are smaller than 1 pixel. Due to the load applied, the surface of the specimen located above the holes presents an upwards vertical motion, 

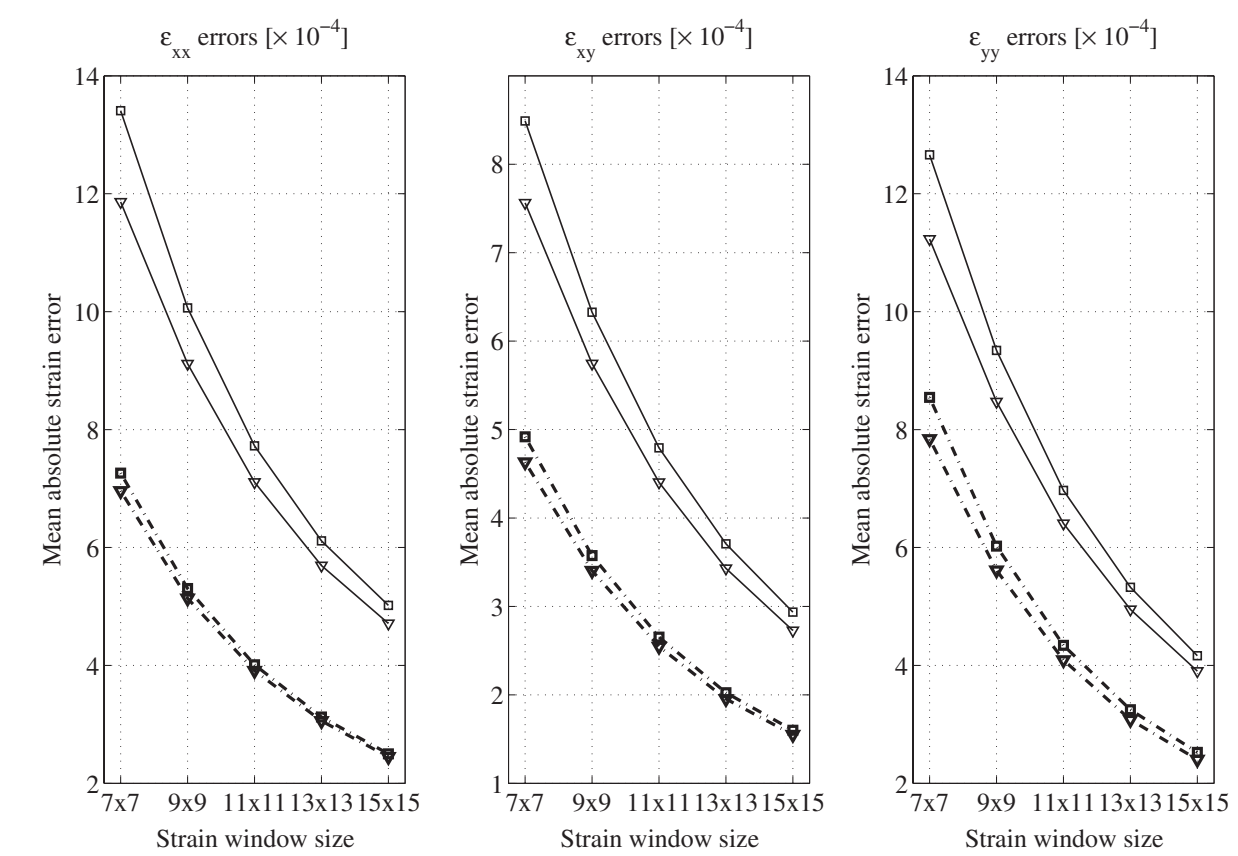

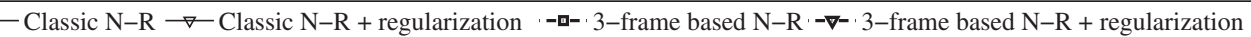

Figure 8. Mean absolute strain errors for the second experiment, $21 \times 21$ pixel subsets.

while the region located below the holes moves very little. The horizontal specimen motion is inwards. Also, where present, horizontal displacements are on average significantly smaller than vertical ones.

In figure 2 , the reference image used in the numerical experiments and the 285th image in the real image sequence are shown. It is evident that the images in the real experiments present smaller speckles and that locally, intensity variations occur at significantly higher frequencies.

In figure 3 , a $50 \times 50$ pixel area inside the first reference image used in the numerical experiments is shown with and without image noise.

\subsection{Experimental results}

The DIC methods employ subsets $21 \times 21,25 \times 25$ and $29 \times$ 29 pixels with a 3 pixel step size. In calculating the strains, strain windows sized $7 \times 7,9 \times 9,11 \times 11,13 \times 13$ and $15 \times 15$ displacements and the pointwise least-squares fitting technique [24] are used. In the regularization term, the regularization strength parameter value is set to 100, regardless of the DIC method. The rest of the parameters remain unchanged with respect to the implementation used in [27]. The three-frame method utilizes all images to calculate the displacements and strains. The results will numerically describe the deformation between any two consecutive images. The classic NewtonRaphson method uses only two images from each of the sequences, namely the reference and first deformed image.

3.2. Strain accuracy analysis. The mean absolute horizontal, shear and vertical strain errors obtained from using subsets sized $21 \times 21,25 \times 25$ and $29 \times 29$ pixels, in the first experiment, are shown in figures 4, 5 and 6, respectively. The first observation that arises from examining results is that the proposed three-frame DIC approach brings accuracy improvements over the classic Newton-Raphson approach regardless of the subset size or strain window. The mean absolute strain errors obtained with the three-frame method are $10 \%-25 \%$ lower than those obtained using the classic DIC method. Regularization also improves accuracy. However, it is more effective for the classic method and low subset and strain sizes. When using $25 \times 25$ and $29 \times 29$ pixel subsets the benefits brought by spatial regularization in the case of the three-frame method are very limited and decrease with increasing strain window size. It is also interesting to note that increasing the subset size lowers the overall strain error only when using small strain windows. It is evident that for each strain, the errors corresponding to the smaller strain windows decrease significantly more than the errors for large strain windows with the subset increase. For example, in the case of the three-frame method (with and without regularization), the minimum errors are around $4 \times 10^{-5}$ for $\epsilon_{x x}, 3 \times 10^{-5}$ for $\epsilon_{x y}$ and $6 \times 10^{-5}$ for $\epsilon_{y y}$, and do not change with the subset size.

Considering the strain errors from figure 4 , the proposed method without regularization presents mean horizontal strain errors between $25 \%$ and $41 \%$ (depending on the strain window size) lower than the classic DIC method without regularization. Similar accuracy improvements in terms of mean strain errors can be reported for the other strains: between $23 \%$ and $38 \%$ for the shear strains and between $18 \%$ and $24 \%$ for the vertical strains.

Although in terms of the mean absolute strain error, using three images improves accuracy by approximately $20 \%$ or more, averaging the strain errors can 'hide' more significant, localized accuracy improvements. Let us look at the theoretical 

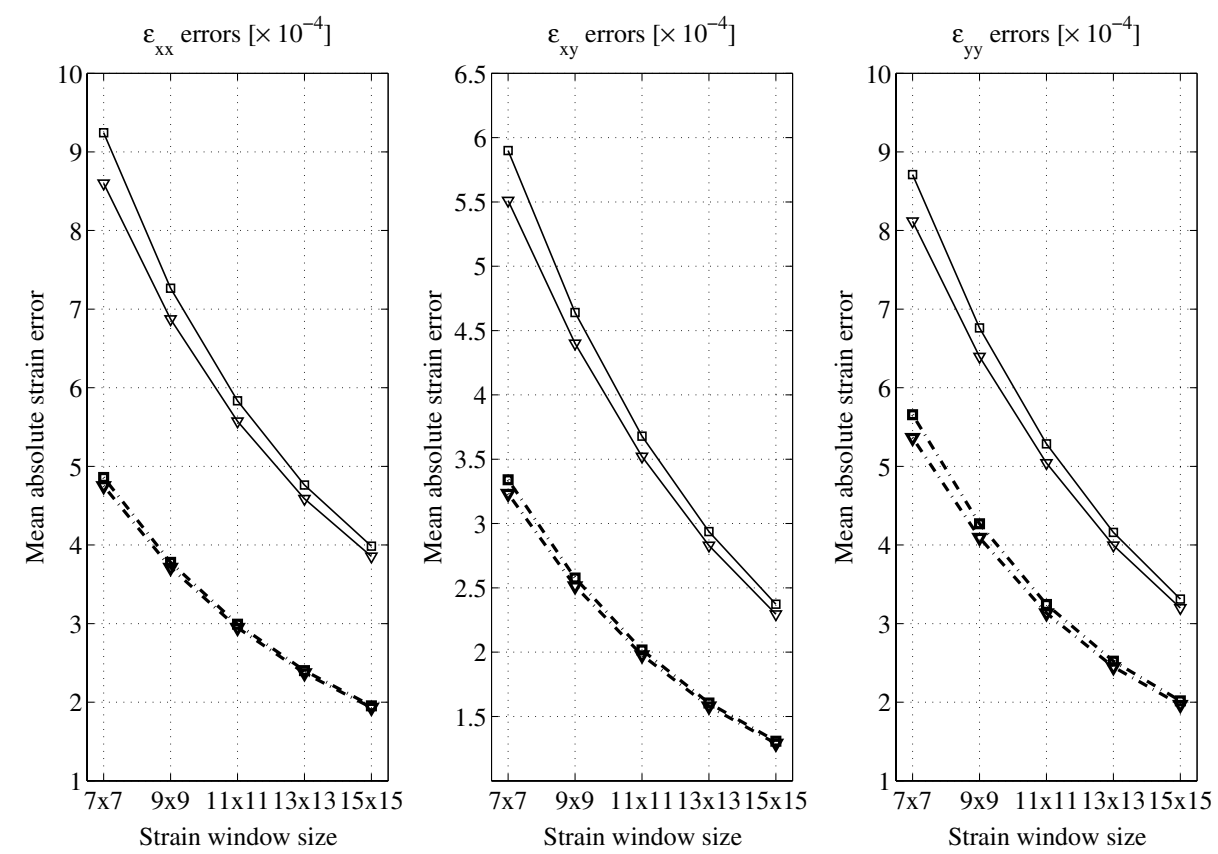

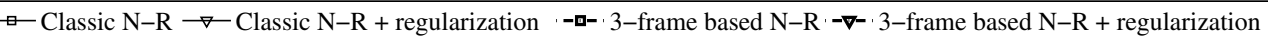

Figure 9. Mean absolute strain errors for the second experiment, $25 \times 25$ pixel subsets.
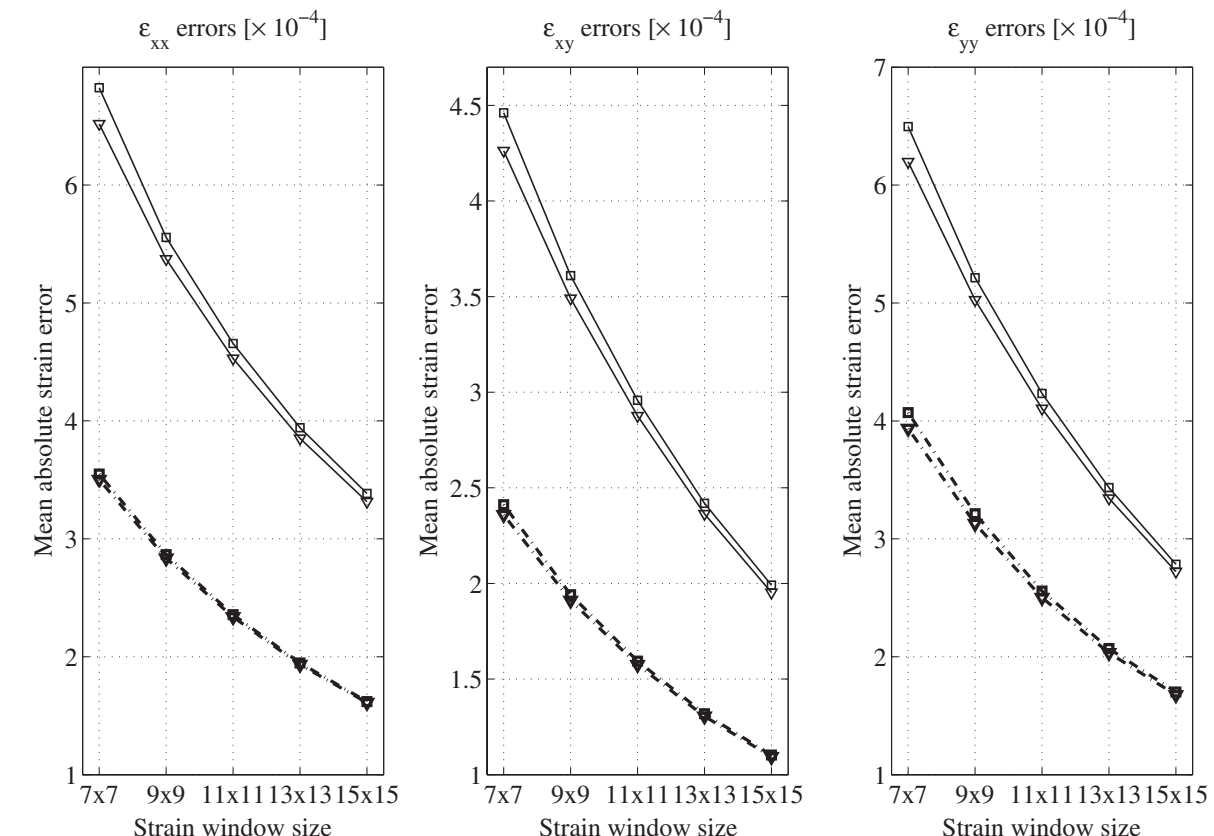

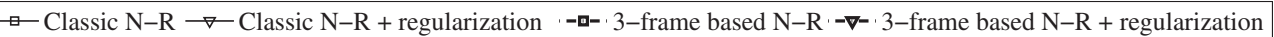

Figure 10. Mean absolute strain errors for the second experiment, $29 \times 29$ pixel subsets.

and some of the calculated shear and vertical strains corresponding to this experiment. The strains are illustrated in figure 7 and were obtained using subset and strain window sizes of $21 \times 21$ pixels and $11 \times 11$ displacements, respectively. Figure 7(a) shows the theoretical strains, figure $7(b)$ the strains calculated using the classic Newton-Raphson DIC method and figure $7(c)$ the strains calculated using the three-frame-based
DIC method, with spatial regularization. The images confirm the numerical results, with the three-frame method clearly showing improvements in strain recovery over the method that used only two images. In the case of the horizontal strains, three areas can clearly be seen (two to the right and one to the lower-left side of the central hole), where the strains calculated with the three-frame method are more accurate than the ones 
$\varepsilon_{\mathrm{xy}}\left[\times 10^{-4}\right]$
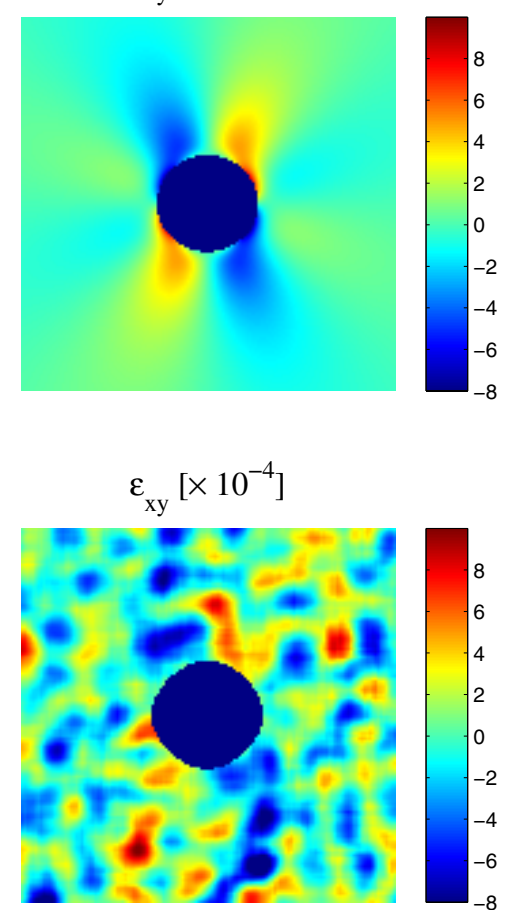

$$
\varepsilon_{x y}\left[\times 10^{-4}\right]
$$

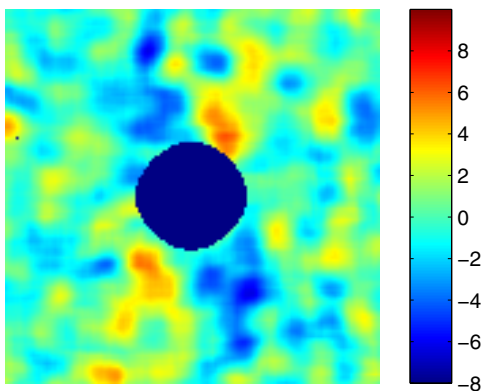

$\varepsilon_{\mathrm{yy}}\left[\times 10^{-3}\right]$

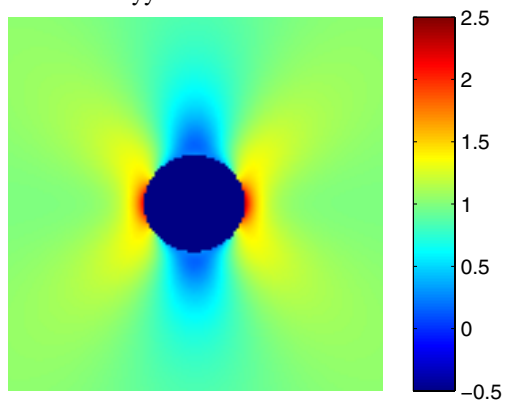

$(a)$

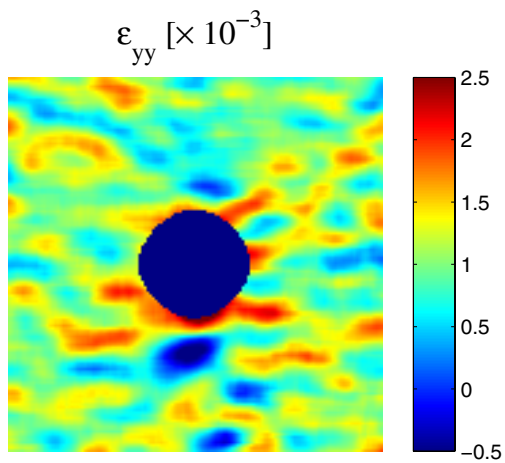

$(b)$

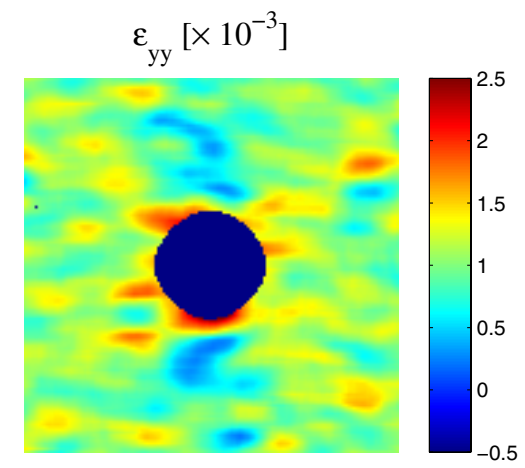

(c)

Figure 11. Shear (left column) and vertical (right column) strains corresponding to the second experiment: $(a)$ theoretical strains; $(b)$ strains calculated through the classic Newton-Raphson DIC method; $(c)$ strains calculated through the three-frame DIC method (using also spatial regularization). The subset size was $21 \times 21$ pixels and the strain window size was $15 \times 15$ displacements.

calculated with the classic Newton-Raphson method. Similar improvements can also be seen when comparing the vertical strain fields. It is also important to note that the three-frame method greatly reduces the occurrence of large errors. In the case of the vertical strains, the errors to the lower-left side of the central hole (the dark red area, figure $7(b)$ ) are up to $100 \%$ larger than the theoretical strains present in those locations, which are approximately $10^{-3}$. In areas where the underlying strains are lower, the percentile errors are even higher. By contrast, in the same region, the errors generated by the threeframe method are approximately $5 \%$ or less of the theoretical values.

The mean absolute horizontal, shear and vertical strain errors for the second experiment considering the three subset sizes are shown in figures 8,9 and 10, respectively. The presence of image noise vastly degrades the mean accuracy of the calculated strains compared to the first experiment. Errors are approximately one order of magnitude higher, regardless of the subset and strain window sizes used. This can be attributed to the fact that small deformations are measured. Small deformations translate into small image intensity differences between the reference and deformed images and, hence, greater sensitivity even to low levels of image noise. The mean strain accuracy gap between the two classes of methods tested is more pronounced, with the threeframe methods clearly producing lower errors. Considering the strain errors shown in figure 8 , the proposed method without regularization presents mean horizontal strain errors between $46 \%$ and $51 \%$ (depending on the strain window size) lower than the classic DIC method without regularization. For the 


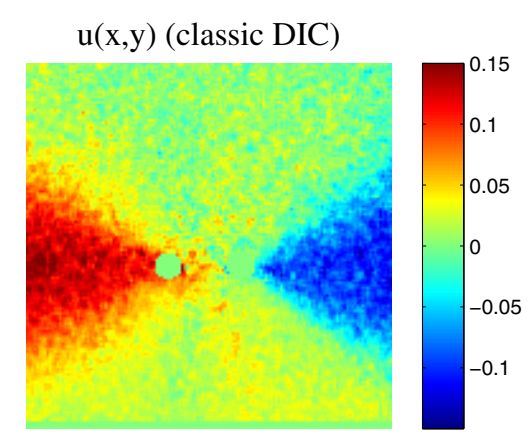

$\mathrm{v}(\mathrm{x}, \mathrm{y})($ classic DIC)

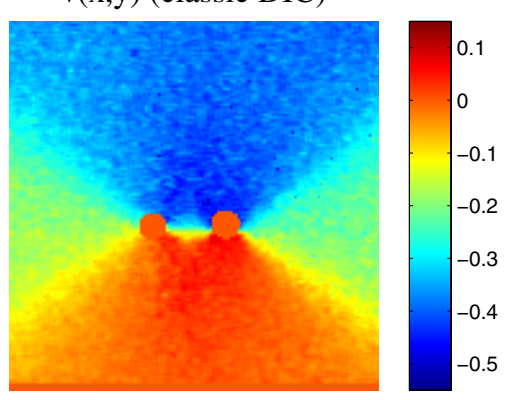

$\mathrm{u}(\mathrm{x}, \mathrm{y})(3-$ frame DIC)

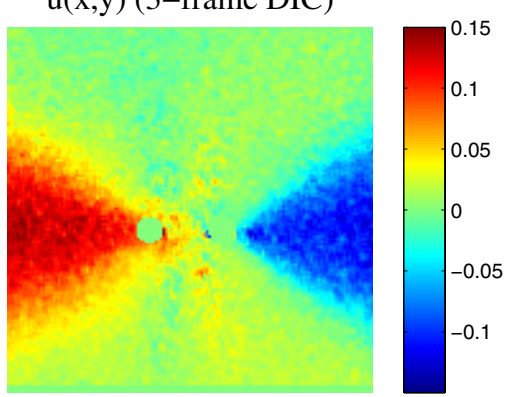

(a)

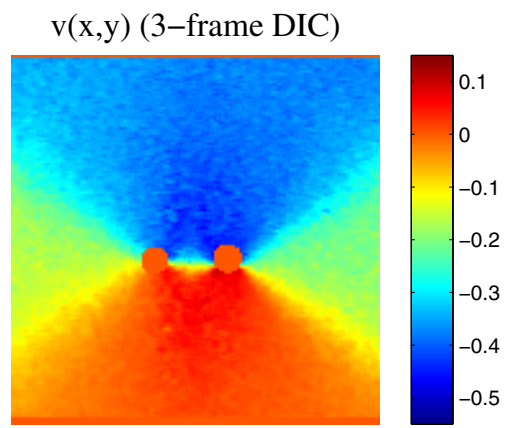

(b)

Figure 12. Third experiment: $(a)$ horizontal and $(b)$ vertical displacements calculated through the classic (left column) and three-frame-based (right column) Newton-Raphson DIC methods. The subset size was $21 \times 21$ pixels.

shear strains, the proposed method's strain errors are between $42 \%$ and $46 \%$ lower than those of the classic method, while for the vertical strains, these are between $32 \%$ and $39 \%$ lower. The impact of regularization is further reduced, with some improvements seen for low subset and strain window sizes.

The theoretical and calculated shear and vertical strains for the second experiment using a subset size of $21 \times 21$ pixels and a strain window size of $15 \times 15$ displacements are illustrated in figure 11. The degradation of the strains due to noise can be clearly seen when comparing the strains with the results from the first experiment. Even though the strains that are shown were calculated with a larger strain window and the dynamic range for displaying results increased, the larger scale of the errors present is evident. The three-frame-based method generates significantly less erroneous strain estimates and manages to create a clearer view of the overall spatial variation of the strains. Nevertheless, large errors remain present in the case of the vertical strains near the edges of the hole. The errors are probably due to the fact that, in those areas, fewer displacement data are used to calculate strains_-displacements corresponding to subsets that partially or fully cover the hole are not used.

\subsection{Real experiment analysis. Figures 12 and 13 show} the displacements and strains corresponding to the third, validation experiment, calculated using the classic and threeframe-based Newton-Raphson DIC methods, both without spatial regularization. The image subset size was $21 \times$ 21 pixels with a subset step of 7 pixels. In calculating the strains, strain windows sized $7 \times 7$ displacements were used. The calculated horizontal displacements are relatively small and do not exceed 0.15 pixels between each two consecutive images in the sequence. These are located on the left and right sides of the two central holes. On the left of the holes the horizontal displacements are positive, indicating a movement from left to right. On the right side displacements are negative, indicating an opposite direction of movement. The polyester film presents vertical displacements of approximately -0.5 pixels above the two holes, signifying an upward movement. Below the holes, displacements are positive and lower than 0.1 pixels.

The directions of the motion are consistent with the material behaviour when subjected to a vertical load oriented upwards. There is little difference concerning the overall spatial variation and typical amplitudes of the displacements between the classic and three-frame based DIC methods. This is a strong indication that the three-frame method deformation assumptions hold for the image sequence in question. However, differences are notable as the classic method produces 'noisier' displacements. The poorer displacement quality becomes clearly visible when looking at the strain fields. Improvements can be seen in the case of the horizontal strains from figure 13(a), in the upper part of the strain fields. In that region, the film sustains predominantly rigid body translations; however, the horizontal strains show localized strains, both positive and negative. It is very unlikely that, given the location and size, these strains are correct. These errors could be reduced if a larger strain window size was used; however, this poses the risk of potentially oversmoothing true strains as well. Vertical strain errors are less visible than horizontal or shear strain ones because the dynamic range of the vertical displacements is larger. Still 


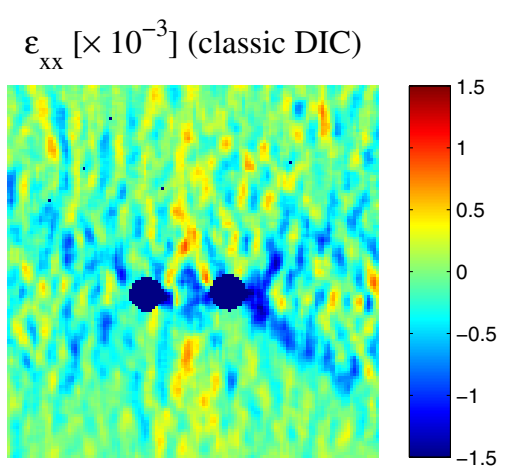

$\varepsilon_{\mathrm{xy}}\left[\times 10^{-3}\right]($ classic DIC $)$

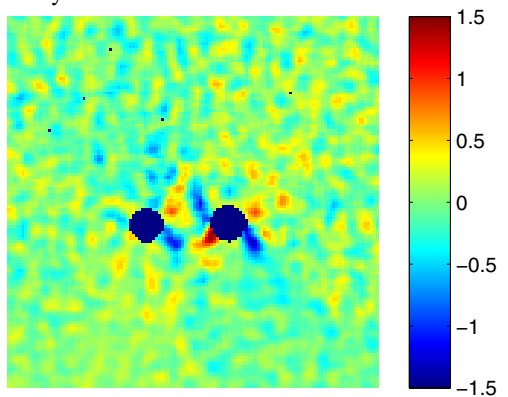

$\varepsilon_{\mathrm{yy}}\left[\times 10^{-3}\right]($ classic DIC)

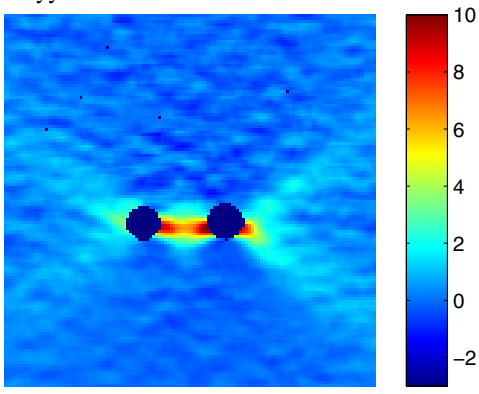

$\varepsilon_{\mathrm{xx}}\left[\times 10^{-3}\right](3-$ frame DIC $)$

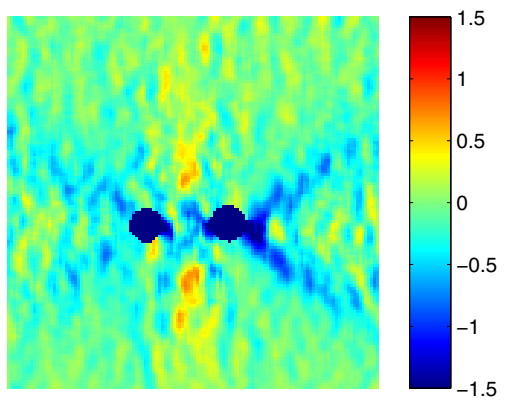

(a)

$\varepsilon_{\mathrm{xy}}\left[\times 10^{-3}\right](3-$ frame DIC $)$

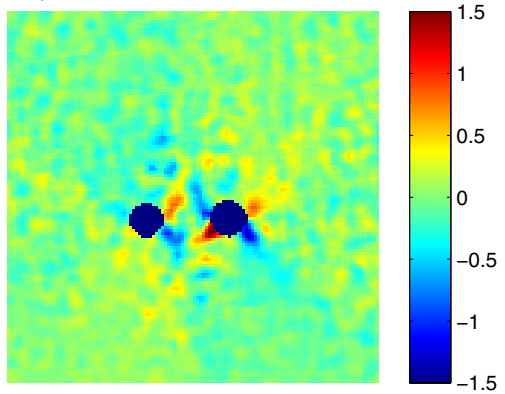

(b)
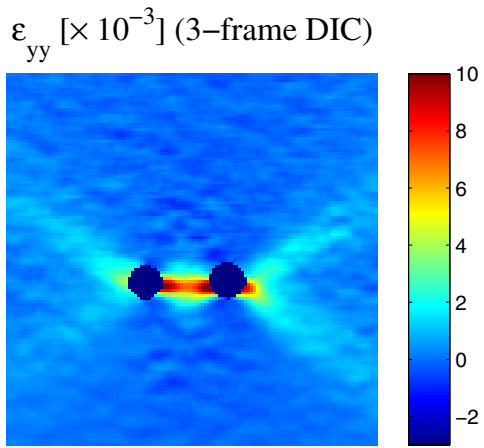

(c)

Figure 13. Third experiment: $(a)$ horizontal, $(b)$ shear and $(c)$ vertical strains calculated through the classic (left column) and three-frame-based (right column) Newton-Raphson DIC methods. The subset size was $21 \times 21$ pixels and the strain window size was $7 \times 7$ displacements.

improvements when using the three-frame DIC method can be seen both above and below the holes. Localized fluctuations where very small vertical strains are present (away from the holes) are reduced. From the results, it is clear that the real experiment confirms that multi-frame methods can bring accuracy improvements over classic DIC methods when measuring small displacements and strains.

3.2. Computational performance. In table 1, speed-related aspects of the three-frame methods are shown, considering the first (numerical) experiment. The image subset size was set at $25 \times 25$ pixels. In the second column of the table, running times relative to the classic Newton-Raphson method are presented. The second column contains the number of iterations performed until convergence was reached. The three-frame methods (with or without regularization) are
Table 1. Speed comparison of the classic and three-frame based Newton-Raphson DIC methods in the first experiment for an image subset size of $25 \times 25$ pixels.

\begin{tabular}{lll}
\hline DIC method & Time $(\%)$ & Iterations \\
\hline Classic N-R & 100 & 30 \\
Classic N-R + regularization & 145.6 & 49 \\
Three-frame based & 326.3 & 37 \\
Three-frame based + regularization & 421.1 & 37 \\
\hline
\end{tabular}

approximately three times slower than the classic ones. We can see that regularization slows the classic method down by $45 \%$ and the three-frame method by $29 \%$ although for the latter case the number of iterations remains the same.

It should be noted that no speed optimizations were implemented for the DIC methods evaluated. The largest 
computational cost for both classic and three-frame methods in the current implementation is associated with the subset interpolation operation. The three-frame method performs three times as many subset interpolation operations as the classic method, which seems to be well correlated with the running times of the methods. In light of recent developments regarding the re-use of interpolation coefficients to speed up computationally DIC methods [32], it is safe to assume that the speed differences between the threeframe method and the classic DIC approach will decrease considerably. If such improvements were implemented, the computational differences would then largely amount to the calculus of the additional Hessian and Jacobian terms and one-time calculation of the additional interpolation coefficients for the third subset. At this stage, it is difficult to draw any final conclusions on the multi-frame method computational complexity; this line of research requires further investigations.

\section{Conclusions}

In this paper, a new Newton-Raphson-based DIC method that uses sequences of three images to calculate displacements and strains has been investigated. The method locally calculates displacements and strains by simultaneously optimizing subset differences across the whole image sequence. To model subset deformations, the method relied on the assumption that the measurable deformation between each two consecutive frames in the sequence varies little. The three-frame DIC method has been evaluated in a total of three experiments, two numerical and one real. The results show that regardless of whether image noise is present or not, the multi-frame approach brought important accuracy improvements. Even more importantly, it allowed the measurement of small strain values in certain regions of the material where with the classic method the magnitude of the errors rendered any accurate measurement impossible. The increases in measurement accuracy come at the cost of increased computational complexity and additional constraints for the experimental setup in order to ensure that the deformations contained in the consecutive images are similar. These drawbacks can be significantly reduced, however, through careful preparation of the experimental setup and efficient implementation techniques, all of which points to the importance of continuing the research on multi-frame strategies into the future.

\section{References}

[1] Peters W H and Ranson W F 1982 Digital imaging techniques in experimental stress analysis Opt. Eng. 21 427-31

[2] Sutton M A, Wolters W J, Peters W H and McNeill S R 1983 Determination of displacements using an improved digital correlation method Image Vis. Comput. 1 133-9

[3] Chu T C, Ranson W F and Sutton M A 1985 Applications of digital-image-correlation techniques to experimental mechanics Exp. Mech. 25 232-44

[4] Sutton M A, Cheng M Q, Peters W H, Chao Y J and McNeill S R 1986 Application of an optimized digital image correlation method to planar deformation analysis Image Vis. Comput. 4 143-50
[5] Shariat H and Price K E 1990 Motion estimation with more than two frames IEEE Trans. Pattern Anal. Mach. Intell. 12 417-34

[6] Hassan Y A, Blanchat T K and Seeley Jr C H 1992 PIV flow visualization using particle tracking techniques Meas. Sci. Technol. 3 633-42

[7] Stiller C and Konrad J 1999 Estimating motion in image sequences IEEE Signal Process. Mag. 16 70-91

[8] Zelnik-Manor L and Irani M 2000 Multi-frame estimation of planar motion IEEE Trans. Pattern Anal. Mach. Intell. 22 1105-16

[9] Irani M 2002 Multi-frame correspondence estimation using subspace constraints Int. J. Comput. Vis. 48 173-94

[10] Farsiu S, Elad M and Milanfar P 2005 Constrained, globally optimal, multi-frame motion estimation Proc. 2005 IEEE Workshop on Statistical Signal Processing pp 1396-401

[11] Feghali R 2005 Multi-frame simultaneous motion estimation and segmentation IEEE Trans. Consum. Electron. $51245-8$

[12] Chen M J, Li G L, Chiang Y Y and Hsu C T 2006 Fast multiframe motion estimation algorithms by motion vector composition for the MPEG-4/AVC/H.264 standard IEEE Trans. Multimedia 8 478-87

[13] Lim H Y, Kassim A A and de With P H N 2008 Predictive 3D search algorithm for multi-frame motion estimation IEEE Trans. Consum. Electron. 54 1938-46

[14] Wang D, Zhang L and Vincent A 2010 Motion-compensated frame rate up-conversion: part I. Fast multi-frame motion estimation IEEE Trans. Broadcast. 56 133-41

[15] Byun J, Choi J and Kim J 2010 A fast multi-reference frame motion estimation algorithm IEEE Trans. Consum. Electron. 56 1911-7

[16] Shi Y B and Wu C K 2008 Fast multi-frame motion estimation algorithm based on H.264 Comput. Eng. 34 218-20

[17] Chen D J, Chiang F-P, Tan Y S and Don H S 1993 Digital speckle-displacement measurement using a complex spectrum method Appl. Opt. 32 1839-49

[18] Bruck H A, McNeill S R, Sutton M A and Peters W H 1989 Digital image correlation using Newton-Raphson method of partial differential correction Exp. Mech. 29 261-7

[19] Vendroux G and Knauss W G 1998 Submicron deformation field measurements: part 2. Improved digital image correlation Exp. Mech. 38 86-92

[20] Lu H and Cary P D 2000 Deformation measurements by digital image correlation: implementation of a second-order displacement gradient Exp. Mech. 40 393-400

[21] Cheng P, Sutton M A, Schreier H W and McNeill S R 2002 Full-field speckle pattern image correlation with B-spline deformation function Exp. Mech. 42 344-52

[22] Zhang J, Jin G, Ma S and Meng L 2003 Application of an improved subpixel registration algorithm on digital speckle correlation measurement Opt. Laser Technol. 35 533-42

[23] Chen Y N, Jin W Q, Zhao L and Li F W 2008 A subpixel motion estimation algorithm based on digital correlation for illumination variant and noise image sequences Optik 120 835-44

[24] Pan B, Asundi A, Xie H and Gao J 2009 Digital image correlation using iterative least squares and pointwise least squares for displacement field and strain field measurements Opt. Lasers Eng. 47 865-74

[25] Pan B, Wang Z Y and Lu Z X 2010 Genuine full-field deformation measurement of an object with complex shape using reliability-guided digital image correlation $O p t$. Express 18 1011-23

[26] Chen J L, Zhang X C, Zhan N and Hu X Y 2010 Deformation measurement across crack using two-step 
extended digital image correlation method Opt. Lasers Eng. 48 1126-31

[27] Cofaru C, Philips W and Van Paepegem W 2010 Improved Newton-Raphson digital image correlation method for full-field displacement and strain calculation Appl. Opt. 49 6472-84

[28] Cofaru C, Philips W and Van Paepegem W 2012 A novel speckle pattern - adaptive digital image correlation approach with robust strain calculation Opt. Lasers Eng. 50 187-98

[29] Timoner S J and Freeman D M 2001 Multi-image gradient-based algorithms for motion estimation Opt. Eng., Bellingham 40 2003-16

[30] Besnard G, Gueŕard S, Roux S and Hild F 2011 A space-time approach in digital image correlation: movie-DIC Opt. Lasers Eng. 49 71-81
[31] Pan B, Xie H and Wang Z 2010 Equivalence of digital image correlation criteria for pattern matching Appl. Opt. 49 5501-9

[32] Pan B and Li K 2011 A fast digital image correlation method for deformation measurement Opt. Lasers Eng. 49 841-7

[33] Cofaru C, Philips W and Van Paepegem W 2010 Evaluation of digital image correlation techniques using realistic ground truth speckle images Meas. Sci. Technol. 21055102

[34] Van Paepegem W, Shulev A A, Roussev I R, De Pauw S and Degrieck J 2009 Study of the deformation characteristics of window security film by digital image correlation techniques Opt. Lasers Eng. 47 390-7

[35] Cárdenas-García J F and Verhaegh J J E 1999 Catalogue of moiré fringes for a bi-axially-loaded infinite plate with a hole Mech. Reas. Commun. 26 641-8 